\title{
ОПЫТ ОБСЛЕДОВАНИЯ МОЛОЧНЫХ ЖЕЛЕЗ У ЖЕНЩИН С СОЧЕТАННОЙ ПАТОЛОГИЕЙ ЩИТОВИДНОЙ ЖЕЛЕЗЫ
}

Геращенко Я.Л.

\author{
Международный медицинский чентр «Согаз», Санкт-Петербург
}

В статье рассмотрена структура заболеваний молочных желез, у пациенток с патологией щитовидной железы. Особенности терапии выявленных состояний.

ЦЕЛЬ: представить данные о структуре заболеваний молочных желез в группе пациенток с патологией щитовидной железы. Отметить особенности диагностической и лечебной тактики.

АКТУАЛЬНОСТЬ: на современном этапе основная проблема рака молочной железы (РМЖ) состоит не только в усовершенствовании методов лечения, но и в поиске оптимальных путей его ранней и точной диагностики [1], а также лечении мастопатии.

Немаловажную роль в морфогенезе и функциональной дифференцировке эпителиальных клеток молочной железы, играют гормоны щитовидной железы [2,3,6,7]. Несмотря на то, что большинство исследований, посвященных изучению сочетанной патологии щитовидной и молочной желез, акцентирует внимание только на проблеме рака молочной железы,оценка влияния на доброкачественные изменения ткани молочной железы, до сих пор представляет научный интерес $[4,5,8]$.

МАТЕРИАЛЫ И МЕТОДЫ: ретроспективно проанализированы МК 427 пациенток за период с 03.2018 по 12.2019 гг. Количество женщин в группе А (до 50 лет) составило 128 человек. Количество женщин в группе В (старше 50 лет) составило 299 человек. Методы обследования: анамнестический, осмотр, пальпация, ультразвуковое исследование (УЗИ) молочных желез (МЖ), маммография (МГ), магнитно-резонансная томография (МРТ). Критерии оценки наличия или отсутствия патологии МЖ основывался на анализе жалоб, ультразвуковых (УЗ), МГ, МРТ и пальпаторных данных о толщине, плотности, размерах и соотношении тканей. Средний возраст всех обследованных женщин, включенных в исследование 43,6 года. Минимальный возраст составил 17 лет, максимальный 85 лет. Женщинам старше 35 лет выполняли МГ и УЗИ МЖ, младше 35 лет выполнялось УзИ МЖ. По показаниям выполняли МРТ МЖ с внутривенным контрастированием. Все исследования МЖ у женщин с регулярным менструальным циклом проводили с 5 по 10 день цикла. Регистрация данных в электронной МК и анализ показателей проводились в «медицинской информационной системе «МЕДИАЛОГ», версия 2.5 (ООО «Пост Модерн Текнолоджи»). Статистические данные представлены в виде абсолютных цифр и процентного соотношения.

PЕзУЛЬТАТЫ: в группе из 128 женщин старше 50 лет у 16 (12,5\%) в анамнезе установлен диагноз узловой зоб. У 2х (1,5\%) диффузный зоб. У 6 (4,7\%) аутоиммунный тиреоидит.

Из 16 пациенток с диагнозом узловой зоб у 6 (37,5\%) диагностирована кистозная мастопатия (КМ), 3 женщинам с лечебной целью назначен препарат Мастодинон, 3 женщинам назначен Прожестожель, у 1 пациентки (6,25\%) диагностирована фиброаденома (ФА) назначен Индинол форто. у 9 (56,2\%) женщин патологии не выявлено.

Из 2х пациенток с диагнозом диффузный зоб, у 1 пациентки диагностирована ФА, назначен препарат Индинол форто, 1 пациентка направлена в онкологический диспансер.

Из 6 пациенток с диагнозом аутоиммунный тиреоидит, у 1(16,6\%) пациентки выявлен локализованный фиброаденоз, выбрана тактика наблюдения, у 1 (16,6\%) пациентки КМ назначен препарат Мастодинон, у 4x (66,6\%) пациенток патологии не выявлено.

Из 299 женщин группы младше 50 лет, у 19 (6,3\%) из них узловой зоб, у 3 (1\%) диффузный зоб, у 8 (2,7\%) аутоиммунный тиреоидит (АИТ).

Из 19 пациенток с диагнозом узловой зоб, у 11(57,8\%) диагностирована КМ, 6 (31,6\%) женщинам назначен Циклодинон, 5 (26,3\%) женщинам назначен Прожестожель, у 2 (10\%) пациенток диагностирована ФА, назначен Индинол форто, у 1 (5\%) пациентки - вариант нормы, 1 (5\%) женщина направлена на обследование в онкодиспансер, 4 (21\%) женщинам избрана тактика динамического наблюдения. 
Из 3 пациенток с диагнозом диффузный зоб, у 2 (66,7\%) пациенток диагностирована КМ, назначен Циклодинон, у 1 пациентки (33,3\%) диагностирована ФА, назначен Индинол форто.

В группе из 8 пациенток с диагнозом АИТ, у одной пациентки (12,5\%) нормальное строение ткани МЖ, у 5 (62,5\%) диагностирована КМ, троим (37,5\%) назначен Циклодинон, двоим (25\%)- Прожестожель, у одной женщины (12,5\%) диагностирована ФА, ей назначен Индинол форто, одна пациентка (12,5\%) направлена на дообследование в онкодиспансер.

Выводы: патология щитовидной железы является значимой, но несущественной в развитии заболеваний МЖ. Лечение заболеваний МЖ должно проводиться по стандартным алгоритмам и дополняться коррекцией нарушений функции щЖ. Выделение данной группы женщин в отдельный поток нецелесообразно, однако изучение функции ЩЖ важно в алгоритмах маммологического обследования.

КЛЮЧЕВЫЕ СЛОВА: обследование молочных желез, маммолог, заболевания щитовидной железы. 